Chinese Journal of Organic Chemistry

\title{
4-烷基-6-芳基-1,3-噻嗪的合成与生物活性
}

\author{
李 婉 ${ }^{a}$ 胡艾希*, $a$ 刘艾林 ${ }^{*}, b \quad$ 彭俊梅 ${ }^{a}$ 夏 林 ${ }^{a} \quad$ 谭卫清 ${ }^{a}$ \\ ( ${ }^{a}$ 湖南大学化学化工学院 长沙 410082) \\ $\left({ }^{b}\right.$ 中国医学科学院药物研究所 北京 100050)
}

\begin{abstract}
摘要 以芳香醛为原料, 经缩合、环化等合成了 16 种 1,3-噻嗪化合物 $\mathbf{1 a} \sim \mathbf{1 p}$, 并对其进行了体外神经氨酸酶(NA)和 5-差色胺转运体(SERT) 抑制活性测试, 测试结果显示部分化合物对神经氨酸酶和 5-着色胺转运体有抑制活性. 其中化 合物 $1 \mathrm{i}$ 在 $40 \mu \mathrm{g} / \mathrm{mL}$ 浓度下对神经氨酸酶的抑制率达 $68.91 \%, 10 \mu \mathrm{g} / \mathrm{mL}$ 浓度下, 1 d, 1 和 1 f 对 5 -羊色色胺转运体的抑制率 分别为 $99.5 \%, 99.6 \%$ 和 $99.8 \%$.
\end{abstract}

关键词 合成; 1,3-噻嗪; 神经氨酸酶抑制剂; 5-羊色色胺转运体抑制剂

\section{Synthesis and Biological Activities of 4-Alkyl-6-aryl-1,3-thiazines}

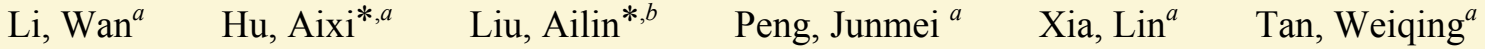 \\ ( ${ }^{a}$ College of Chemistry and Chemical Engineering, Hunan University, Changsha 410082) \\ ( ${ }^{b}$ Institute of Materia Medica, Chinese Academy of Medical Sciences and Peking Union Medical College, Beijing 100050)
}

\begin{abstract}
A series of 1,3-thiazine derivatives $\mathbf{1 a} \sim \mathbf{1 p}$ were designed and synthesized by the reaction of aromatic aldehyde via condensation and cyclization, and their inhibitory activity of nueraminidase (NA) and serotonin reuptake (SERT) were evaluated in vitro. The pharmacological results showed that compound $\mathbf{1 i}$ exhibits good activity of NA with the inhibitory rate of $68.91 \%$ at the concentration of $40 \mu \mathrm{g} / \mathrm{mL}$, which is similar to oseltamivir (inhibitory rate is $72.6 \%$ ), moreover, at the concentration of $10.0 \mu \mathrm{g} / \mathrm{mL}$, compounds $\mathbf{1 d}, \mathbf{1 e}$ and $\mathbf{1 f}$ exhibit good serotonin reuptake inhibitory activity with inhibitory rates of $99.5 \%, 99.6 \%$ and $99.8 \%$, respectively.
\end{abstract}

Keywords synthesis; 1,3-thiazine; neuraminidase inhibitors; serotonin transportor inhibitors

流行性感冒是一种由流感病毒引起的急性呼吸道 感染疾病, 具有传染性强、流行面广、发病率高等特点, 能感染人类和其他动物. 近年来流行的 H5N1, H1N1 使 得流感成为全球性的首要卫生问题之一. 神经氨酸酶 (NA)是流感病毒表面的一种糖蛋白, 在流感病毒的复 制和感染中起着重要作用, 因其在流感病毒变异过程中 具有相对的保守性而成为抗流感病毒药物研究的重要 靶标 ${ }^{[1 \sim 3]}$. 目前上市的 NA 抑制剂有奥斯米韦 (Oseltamivir), 扎那米韦(Zanamivir) 以及帕拉米韦(Peramivir). 奥斯米 韦是第一个口服有效的 NA 抑制剂, 商品名 “达菲”, 但 随着奥斯米韦的广泛使用，世界各地陆续出现了其耐药 病毒株 ${ }^{[4,5]}$. 扎那米韦活性很好, 但生物利用度低, 只能 口服吸入或滴鼻给药, 且价格昂贵, 合成路线复杂较难 获得 ${ }^{[6,7]}$. 因此, 研究开发新的 NA 抑制剂显得尤为重要.
抑郁症是目前被医学界公认的一种常见精神疾病. 2002 年, 世界卫生组织将抑有症确定为世界第四大疾 病，仅仅过了 10 年时间，抑郁症现已上升为世界第二大 疾病. 目前, 中国抑郁症的患病率约为 $6 \%$, 抑郁症患者 估计有 3600 万人. 抗抑郁药按其作用靶点来分析, 可分 为去甲肾上腺素重摄取抑制剂(新三环类抗抑郁药), 单 胺氧化酶抑制剂和 5 -差色色胺 (5-HT)重摄取抑制剂 ${ }^{[8]}$. 去 甲肾上腺素重摄取抑制剂显效慢, 大多数患者在使用一 周后才能观察到疗效. 单胺氧化酶抑制剂药物含肼的结 构复杂，毒性大，副作用多，限制了临床上的应用. 5-HT 重摄取抑制剂选择性强, 安全性大, 副作用低, 口 服吸收好, 生物利用度高, 是临床应用最多最广的一线 抗抑郁药 ${ }^{[9 \sim 12]}$.

1,3-噻溙类化合物作为重要的杂环化合物, 广泛应

\footnotetext{
*E-mail: axhu@hnu.edu.cn; liuailin@imm.ac.cn

Received December 17, 2012; revised March 2, 2013; published online March 6, 2013

Project supported by the Ph.D. Programs Foundation of Ministry of Education of China (No. 20040532002).

国家教育部博士点专项基金(No. 20040532002)资助项目.
} 
用于医药中, 可作为抗高血压、利尿、抗精神病和抗过 敏等药物使用, 最近研究报道其还有麻醉和抗疲劳等功 效 ${ }^{[13 \sim 18]}$. 鉴于 1,3 -噻嗪类化合物的广泛生物活性, 本文 以 1,3 -噻嗪环为母核, 针对神经氨酸酶组一中新发现的 430-loop 和 150-loop ${ }^{[19 \sim 21]}$, 设计合成了一系列 4-叔丁基6-芳基-2-氨基-1,3-噻嗪化合物. 以噻嗪环为连接基团, 疏水性芳环和叔丁基分别与 430-loop 和 150-loop 作用. 另外, 1,3-噻嗪母环上的氨基也能与唾液酸(SA)活性位 点中极性氨基酸结合. 本文以芳香醛为原料, 经缩合、 环化反应合成了 16 个 1,3 - 噻嗪类化合物 $(\mathbf{1 a} \sim \mathbf{1 p}$, Scheme 1), 对其进行了体外 NA (H1N1)抑制活性测试. 同时发现该系列化合物对 5-羟色胺转运体(SERT)也有 很好的抑制活性.

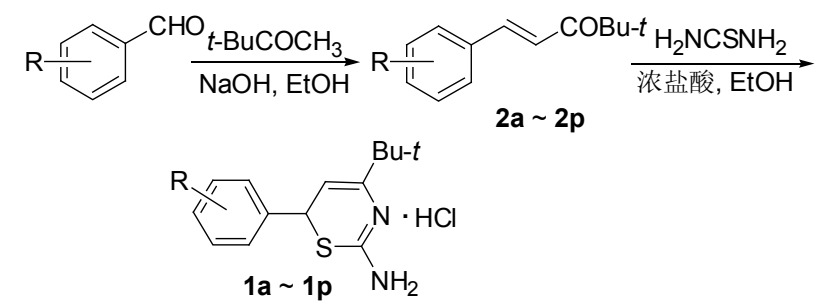

a: $\mathrm{R}=3-\mathrm{NO}_{2} ; \quad$ b: $\mathrm{R}=3-\mathrm{C}_{6} \mathrm{H}_{5} \mathrm{CH}_{2} \mathrm{O}-4-\mathrm{MeO} ; \quad$ c: $\mathrm{R}=2-\mathrm{Cl}-5-\mathrm{NO}_{2}$;

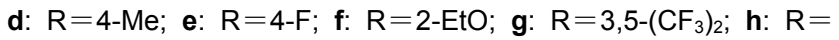
$4-\mathrm{NO}_{2} ; \mathbf{i}: \mathrm{R}=3,4-(\mathrm{MeO})_{2} ; \mathrm{j}: \mathrm{R}=4-\mathrm{MeO} ; \mathbf{k}: \mathrm{R}=2-\mathrm{Cl} ; \mathrm{I}: \mathrm{R}=4-\mathrm{Cl} ; \mathbf{m}$ : $\mathrm{R}=2,4-\mathrm{Cl}_{2} ; \mathbf{n}: \mathrm{R}=2-\mathrm{MeO} ; \mathbf{o}: \mathrm{R}=3-\mathrm{CF}_{3} ; \mathbf{p}: \mathrm{R}=\mathrm{H}$

\section{Scheme 1}

\section{1 结果与讨论}

以结构简单的芳香醛和频哪酮为原料, 经 Adol 缩
合得到了中间体 4,4-二甲基-1-芳基-1-烯基-3-戊酮(2a $\mathbf{2 p})^{[22]}$, 反应收率较高 $(79 \% \sim 95 \%) . \mathbf{2 a} \sim \mathbf{2 p}$ 与硫腿经 “Atwal” 反应，环合得到 4-叔丁基-6-芳基-2-氨基-1,3噻嗪盐酸盐 $1 \mathbf{a} \sim 1 \mathbf{p}$, 用 ${ }^{1} \mathrm{H} N \mathrm{NMR}$ 和 MS 确认了化合物结 构, 并对化合物 11 和 21 进行了单晶 $X$ 射线衍射 ${ }^{[23,24]}$.

化合物 1a 1p 对 NA 和 SERT 的体外抑制活性见 表 1. 从表 1 可以看出, 在 $40 \mu \mathrm{g} / \mathrm{mL}$ 的浓度下，化合物 1a 1p 中部分化合物对 NA 的抑制率较高. 其中, 6-位 芳环上带供电子基团的化合物 $1 \mathbf{f}, \mathbf{1 j}, \mathbf{1 i}$ 和 $1 \mathrm{n}$ 的活性要 高于带吸电子基团的化合物 1a, 1g, $1 \mathrm{k}$ 和 11; 6-位芳环上 带甲氧基的化合物的活性要高于其它化合物. 化合物 $\mathbf{~ i ~}$ 的活性最好，其抑制率和 $\mathrm{IC}_{50}$ 分别为 $68.91 \%$ 和 23.54 $\mu \mathrm{mol} / \mathrm{mL}$ (同等浓度下阳性对照物奥斯米韦的抑制率为 72.6\%). 推其原因噻嗪环 6-位苯环上的 3,4-位甲氧基使 得化合物疏水作用增强, 与 430-loop 和 150-loop 结合较 好.

化合物 1a 1p 中部分化合物对 SERT 抑制活性较 好. 其中，6-位芳环上邻位和对位取代的化合物的活性 高于间位取代的化合物；6-位芳环上间位取代的化合物 活性高于 6-位芳环上二取代的化合物. 在 $10.0 \mu \mathrm{g} / \mathrm{mL}$ 的 浓度下, 1d, 1e 和 1f 抑制率分别为 99.5\%, 99.6\%和 $99.8 \%$.

\section{2 结论}

芳香醛与频哪酩缩合得到了中间体 4,4-二甲基-1芳基-1-烯基-3-戊酥(2a～2p)，收率在 79\%～95\%之间.

表 1 化合物 $1 \mathrm{a} \sim 1 \mathrm{p}$ 对 NA 和 SERT 的抑制活性

Table 1 The inhibitory activities of compounds $\mathbf{1 a} \sim \mathbf{1 p}$ to NA and SERT

\begin{tabular}{|c|c|c|c|c|}
\hline \multirow{2}{*}{ Compd. } & \multirow{2}{*}{$\mathrm{R}$} & \multicolumn{2}{|l|}{ NA } & \multirow{2}{*}{$\begin{array}{c}\text { SERT } \\
\text { Inhibitory rate } / \%(10.0 \mu \mathrm{g} / \mathrm{mL})\end{array}$} \\
\hline & & Inhibitory rate $/ \%(40 \mu \mathrm{g} / \mathrm{mL})$ & $\mathrm{IC}_{50} /\left(\mu \mathrm{g} \cdot \mathrm{mL}^{-1}\right)$ & \\
\hline 1a & $3-\mathrm{NO}_{2}$ & 11.7 & & 50.2 \\
\hline $1 \mathbf{b}$ & $3-\mathrm{C}_{6} \mathrm{H}_{5} \mathrm{CH}_{2} \mathrm{O}-4-\mathrm{MeO}$ & 4.2 & & -0.2 \\
\hline $1 \mathrm{c}$ & $2-\mathrm{Cl}-5-\mathrm{NO}_{2}$ & 6.0 & & -21.0 \\
\hline 1d & $4-\mathrm{Me}$ & 0.7 & & 99.5 \\
\hline $1 e$ & $4-\mathrm{F}$ & -4.2 & & 99.6 \\
\hline 1f & 2-EtO & 13.8 & & 99.8 \\
\hline $1 g$ & $3,5-\left(\mathrm{CF}_{3}\right)_{2}$ & -2.0 & & 21.4 \\
\hline $1 \mathrm{~h}$ & $4-\mathrm{NO}_{2}$ & -0.5 & & 29.3 \\
\hline $1 \mathbf{i}$ & $3,4-(\mathrm{MeO})_{2}$ & 68.9 & 23.54 & 17.2 \\
\hline $\mathbf{1 j}$ & $4-\mathrm{MeO}$ & 9.9 & & 85.9 \\
\hline $1 \mathrm{k}$ & $2-\mathrm{Cl}$ & 2.6 & & 37.4 \\
\hline 11 & $4-\mathrm{Cl}$ & -0.9 & & 16.1 \\
\hline $1 \mathrm{~m}$ & $2,4-\mathrm{Cl}_{2}$ & 19.4 & & 24.8 \\
\hline 1n & 2-MeO & 31.5 & & 16.2 \\
\hline 10 & $3-\mathrm{CF}_{3}$ & 7.8 & & 20.4 \\
\hline $1 p$ & $\mathrm{H}$ & 17.2 & & 8.5 \\
\hline Oseltamivir & & 72.6 & 0.434 & \\
\hline Fluoxetine & & & & $55.0(2.3 \mu \mathrm{g} / \mathrm{mL})$ \\
\hline
\end{tabular}


$\mathbf{2 a} \sim 2 \mathbf{p}$ 与硫腿发生 “Atwal” 反应, 得到目标化合 物 1a 1p, 收率在 30\% 92\%之间, 反应条件温和, 操 作简单。

合成的 4-叔丁基-6-芳基-2-氨基-1,3-噻嗪盐酸盐 1a 1p. 经体外 NA 抑制活性测试, 发现部分化合物对 $\mathrm{NA}$ 有良好的抑制活性, 在 $40 \mu \mathrm{g} / \mathrm{mL}$ 的浓度下, 化合物 $1 \mathrm{i}$ 活性最好(抑制率为 $68.91 \%$ ).

化合物 1a $\sim 1 p$ 中部分化合物对 SERT 的抑制活性 较好; 在 $10.0 \mu \mathrm{g} / \mathrm{mL}$ 的浓度下, $1 \mathbf{1 d} \sim \mathbf{1 f}$ 抑制率分别为 $99.5 \%, 99.6 \%$ 和 $99.8 \%$. 活性好的化合物值得进一步的 研究.

\section{3 实验部分}

\section{1 仪器与试剂}

RY-1 熔点仪未校正(天津天分仪器厂); 薄层层析硅 胶板(烟台市芝罟黄务硅胶开发试剂厂); 三用紫外仪(上 海安亭仪器厂)显色; VARIAN INOVA-400 核磁共振仪 (美国), ${ }^{1} \mathrm{H}$ NMR 核磁共振频率为 $399.970 \mathrm{MHz}, \mathrm{TMS}$ 为 内标; VARIO EL III 元素分析仪(德国); Brucker SMART APEX-CCD 探测器 X 射线单晶衍射仪 (德国); 连续光谱 酶标测试仪(spectramax M5) (美国). 溶剂和试剂均为市 售分析纯或化学纯.

\section{2 化合物的合成}

3.2.1 4,4-二甲基-1-芳基-1-烯基-3-戊酮 $(\mathbf{2 a} \sim 2 \mathbf{p})$ 的 合成

按文献[22]方法合成化合物 2a 2 $\mathbf{p}$, 收率为 79\% $95 \%$.

3.2.2 4-叔丁基-6-芳基-2-氨基-1,3-噻嗪盐酸盐(1a 1p)的合成

4-叔丁基-6-(3-硝基苯基)-2-氨基-1,3-噻嗪盐酸盐 (1a)的合成: $3.25 \mathrm{~g}(0.015 \mathrm{mmol}$ ) 4,4-二甲基-(3-硝基苯 基)-1-烯基-3-戊酮(2a), $1.37 \mathrm{~g}(0.018 \mathrm{~mol}$ )硫脲及 $50 \mathrm{~mL}$ 乙醇, $1.5 \mathrm{~mL}$ 浓盐酸催化反应, TLC 监测反应, 回流反应 $8 \mathrm{~h}$, 减压蒸去大部分溶剂, 浓缩物置冰箱中, 析出黄色 固体, 抽滤, 干燥得 1a, 收率 55\%. m.p. 137 $138{ }^{\circ} \mathrm{C} ;{ }^{1} \mathrm{H}$ NMR $\left(400 \mathrm{MHz}, \mathrm{CDCl}_{3}\right) \delta: 1.23\left(\mathrm{~s}, 9 \mathrm{H}, \mathrm{CH}_{3} \times 3\right), 4.89$ (d, $J=6.8 \mathrm{~Hz}, 1 \mathrm{H}$, 噻嗪环 $5-\mathrm{H}), 5.35(\mathrm{~d}, J=6.8 \mathrm{~Hz}, 1 \mathrm{H}$, 噻 嗪环 6-H), 7.51 8.16 (m, 4H, $\left.\mathrm{C}_{6} \mathrm{H}_{4}\right), 9.53,10.29(2 \mathrm{~s}, 2 \mathrm{H}$, $\left.\mathrm{NH}_{2}\right), 11.50\left(\mathrm{~s}, 1 \mathrm{H}, \mathrm{N}^{+} \mathrm{H}\right)$; EI-MS $m / z$ : $291\left(\mathrm{M}^{+}\right)$. Anal. calcd for $\mathrm{C}_{14} \mathrm{H}_{18} \mathrm{ClN}_{3} \mathrm{O}_{2} \mathrm{~S}: \mathrm{C} 51.29, \mathrm{H} 5.53, \mathrm{~N}$ 12.82; found C 51.19, H 5.55, N 12.79 .

化合物 1b 1e 合成方法同 1a; $1 \mathrm{f} \sim 1 \mathrm{p}$ 按中国授权 专利 $[25,26]$ 方法合成. $1 \mathrm{~b} \sim \mathbf{1 p}$ 合成的反应时间、收率、 熔点和谱图数据如下:

4-叔丁基-6-(3-芐氧苯基-4-甲氧基)-2-氨基-1,3-噻嗪 盐酸盐(1b): 反应 $7 \mathrm{~h}$, 收率 $78 \%$. m.p. $145 \sim 146{ }^{\circ} \mathrm{C} ;{ }^{1} \mathrm{H}$ NMR (400 MHz, $\left.\mathrm{CDCl}_{3}\right) \delta: 1.30\left(\mathrm{~s}, 9 \mathrm{H}, \mathrm{CH}_{3} \times 3\right), 3.89$ (s, $\left.3 \mathrm{H}, \mathrm{OCH}_{3}\right), 4.92(\mathrm{q}, J=4.4 \mathrm{~Hz}, 1 \mathrm{H}$, 噻嗪环 $5-\mathrm{H}), 5.16$ (s, $2 \mathrm{H}, \mathrm{CH}_{2}$ ), 5.20 (q, $J=4.4 \mathrm{~Hz}, 1 \mathrm{H}$, 噻嗪环 6-H), 6.82 $6.83\left(\mathrm{~m}, 3 \mathrm{H}, \mathrm{C}_{6} \mathrm{H}_{3}\right), 7.29 \sim 7.43\left(\mathrm{~m}, 5 \mathrm{H}, \mathrm{C}_{6} \mathrm{H}_{5}\right), 8.52,10.35$ $\left(2 \mathrm{~s}, 2 \mathrm{H}, \mathrm{NH}_{2}\right), 11.66\left(\mathrm{~s}, 1 \mathrm{H}, \mathrm{N}^{+} \mathrm{H}\right)$. Anal. calcd for $\mathrm{C}_{22} \mathrm{H}_{27} \mathrm{ClN}_{2} \mathrm{O}_{2} \mathrm{~S}$ : C 63.07, H 6.50, N 6.69; found C 63.17, $\mathrm{H} 6.55, \mathrm{~N} 6.65$..

4-叔丁基-6-(2-氯-5-硝基苯基)-2-氨基-1,3-噻嗪盐酸 盐(1c): 反应 8 h, 收率 $80 \%$. m.p. $248 \sim 250{ }^{\circ} \mathrm{C} ;{ }^{1} \mathrm{H}$ NMR $\left(400 \mathrm{MHz}, \mathrm{CDCl}_{3}\right) \delta: 1.41\left(\mathrm{~s}, 9 \mathrm{H}, \mathrm{CH}_{3} \times 3\right), 5.20[\mathrm{~s}, 2 \mathrm{H}$, 噻嗪环 5,6- $\left.\mathrm{H}_{2}\right], 7.00\left(\mathrm{q}, J=9.2 \mathrm{~Hz}, 1 \mathrm{H}, \mathrm{C}_{6} \mathrm{H}_{3}-3-\mathrm{H}\right), 8.02$ (s, $\left.1 \mathrm{H}, \mathrm{C}_{6} \mathrm{H}_{3}-6-\mathrm{H}\right), 8.25$ (q, $\left.J=9.2 \mathrm{~Hz}, 1 \mathrm{H}, \mathrm{C}_{6} \mathrm{H}_{3}-4-\mathrm{H}\right)$, 10.72, $11.93\left(2 \mathrm{~s}, 2 \mathrm{H}, \mathrm{NH}_{2}\right)$. Anal. calcd for $\mathrm{C}_{14} \mathrm{H}_{17} \mathrm{Cl}_{2-}$ $\mathrm{N}_{3} \mathrm{O}_{2} \mathrm{~S}$ : C 46.42, H 4.73, N 11.60; found C 46.28, H 4.75, $\mathrm{N} 11.56$.

4-叔丁基-6-(4-甲基苯基)-2-氨基-1,3-噻嗪盐酸盐 (1d): 反应 $8 \mathrm{~h}$, 收率 58\%. m.p. 200 201 ${ }^{\circ} \mathrm{C} ;{ }^{1} \mathrm{H}$ NMR $\left(400 \mathrm{MHz}, \mathrm{CDCl}_{3}\right) \delta: 1.28\left(\mathrm{~s}, 9 \mathrm{H}, \mathrm{CH}_{3} \times 3\right), 2.35(\mathrm{~s}, 3 \mathrm{H}$, $\mathrm{CH}_{3}$ ), 4.96 (q, $J=4.4 \mathrm{~Hz}, 1 \mathrm{H}$, 噻嗪环 $5-\mathrm{H}$ ), 5.19 (q, $J=$ $4.4 \mathrm{~Hz}, 1 \mathrm{H}$, 噻嗪环 6- $\mathrm{H}), 7.16 \sim 7.21\left(\mathrm{~m}, 4 \mathrm{H}, \mathrm{C}_{6} \mathrm{H}_{4}\right), 9.16$, $10.15\left(2 \mathrm{~s}, 2 \mathrm{H}, \mathrm{NH}_{2}\right), 11.45$ (s, $\left.1 \mathrm{H}, \mathrm{N}^{+} \mathrm{H}\right)$; EI-MS $m / z: 260$ $\left(\mathrm{M}^{+}\right)$. Anal. calcd for $\mathrm{C}_{15} \mathrm{H}_{21} \mathrm{ClN}_{2} \mathrm{~S}: \mathrm{C}$ 60.69, H 7.13, N 9.44; found C 60.54, H 7.15, N 9.42.

4-叔丁基-6-(4-氟苯基)-2-氨基-1,3-噻嗪盐酸盐(1e): 反应 7 h, 收率 89\%. m.p. $223 \sim 225{ }^{\circ} \mathrm{C} ;{ }^{1} \mathrm{H}$ NMR $(400$ $\left.\mathrm{MHz}, \mathrm{CDCl}_{3}\right) \delta: 1.30\left(\mathrm{~s}, 9 \mathrm{H}, \mathrm{CH}_{3} \times 3\right), 5.03,5.20[2 \mathrm{~s}, 2 \mathrm{H}$, 噻嗪环 5,6- $\mathrm{H}_{2}$ ], $7.05 \sim 7.33\left(\mathrm{~m}, 4 \mathrm{H}, \mathrm{C}_{6} \mathrm{H}_{4}\right), 9.15,10.22$ $\left(2 \mathrm{~s}, 2 \mathrm{H}, \mathrm{NH}_{2}\right), 11.51\left(\mathrm{~s}, 1 \mathrm{H}, \mathrm{N}^{+} \mathrm{H}\right)$. Anal. calcd for $\mathrm{C}_{14} \mathrm{H}_{18} \mathrm{ClFN}_{2} \mathrm{~S}$ : C 55.90, H 6.03, N 9.31; found C 55.75, H $6.05, \mathrm{~N} 9.28$.

4-叔丁基-6-(2-乙氧基苯基)-2-氨基-1,3-噻嗪盐酸盐 (1f): 收率 30\%. m.p. 162 164 ${ }^{\circ} \mathrm{C} ;{ }^{1} \mathrm{H}$ NMR $(400 \mathrm{MHz}$, $\left.\mathrm{CDCl}_{3}\right) \delta: 1.35\left(\mathrm{~s}, 9 \mathrm{H}, \mathrm{CH}_{3} \times 3\right), 1.45 \sim 1.46\left(\mathrm{~m}, 3 \mathrm{H}, \mathrm{CH}_{3}\right)$, $4.07 \sim 4.13\left(\mathrm{~m}, 2 \mathrm{H}, \mathrm{OCH}_{2}\right), 5.17(\mathrm{~d}, J=6.4 \mathrm{~Hz}, 1 \mathrm{H}$, 噻嗪 环 5-H), $5.26(\mathrm{~d}, J=6.4 \mathrm{~Hz}, 1 \mathrm{H}$, 噻嗪环 6-H), $6.88(\mathrm{~d}, J=$ $\left.7.8 \mathrm{~Hz}, 1 \mathrm{H}, \mathrm{C}_{6} \mathrm{H}_{4}-3-\mathrm{H}\right), 7.30$ (t, $\left.J=7.6 \mathrm{~Hz}, 1 \mathrm{H}, \mathrm{C}_{6} \mathrm{H}_{4}-4-\mathrm{H}\right)$, $6.94\left(\mathrm{t}, J=7.8 \mathrm{~Hz}, 1 \mathrm{H}, \mathrm{C}_{6} \mathrm{H}_{4}-5-\mathrm{H}\right), 7.13(\mathrm{~d}, J=7.6 \mathrm{~Hz}, 1 \mathrm{H}$, $\left.\mathrm{C}_{6} \mathrm{H}_{4}-6-\mathrm{H}\right), 7.65,10.45$ (2s, 2H, $\left.\mathrm{NH}_{2}\right), 11.75\left(\mathrm{~s}, 1 \mathrm{H}, \mathrm{N}^{+} \mathrm{H}\right)$; EI-MS $m / z$ : $290\left(\mathrm{M}^{+}\right)$. Anal. calcd for $\mathrm{C}_{16} \mathrm{H}_{23} \mathrm{ClN}_{2} \mathrm{OS}$ : C 58.79, H 7.09, N 8.57; found C 58.69, H 7.12, N 8.55.

4-叔丁基-6-[3,5-二(三氟甲基)苯基]-2-氨基-1,3-噻 溙盐酸盐(1g): 收率 63\%. m.p. 176 178 ${ }^{\circ} \mathrm{C} ;{ }^{1} \mathrm{H}$ NMR $\left(400 \mathrm{MHz}, \mathrm{CDCl}_{3}\right) \delta: 1.27\left(\mathrm{~s}, 9 \mathrm{H}, \mathrm{CH}_{3} \times 3\right), 5.03(\mathrm{~d}, J=$ $5.6 \mathrm{~Hz}, 1 \mathrm{H}$, 噻嗪环 $5-\mathrm{H}), 5.21(\mathrm{~d}, J=5.6 \mathrm{~Hz}, 1 \mathrm{H}$, 噻嗪环 
6-H), $6.85 \sim 6.92\left(\mathrm{~m}, 3 \mathrm{H}, \mathrm{C}_{6} \mathrm{H}_{3}\right), 9.33,10.09(2 \mathrm{~s}, 2 \mathrm{H}$, $\left.\mathrm{NH}_{2}\right), 11.40\left(\mathrm{~s}, 1 \mathrm{H}, \mathrm{N}^{+} \mathrm{H}\right)$. Anal. calcd for $\mathrm{C}_{16} \mathrm{H}_{17} \mathrm{ClF}_{6} \mathrm{~N}_{2} \mathrm{~S}$ : C 45.88, H 4.09, N 6.69; found C 45.74, H 4.07, N 6.68.

4-叔丁基-6-(4-硝基苯基)-2-氨基-1,3-噻嗪盐酸盐 (1h): 收率 93\%. m.p. 198 200 ${ }^{\circ} \mathrm{C} ;{ }^{1} \mathrm{H}$ NMR $(400 \mathrm{MHz}$, $\left.\mathrm{CDCl}_{3}\right) \delta: 1.3\left(\mathrm{~s}, 9 \mathrm{H}, \mathrm{CH}_{3} \times 3\right), 5.19(\mathrm{~d}, J=5.6 \mathrm{~Hz}, 1 \mathrm{H}$, 噻 嗪环 $5-\mathrm{H}), 5.28(\mathrm{~d}, J=5.6 \mathrm{~Hz}, 1 \mathrm{H}$, 噻嗪环 6-H), 7.61 $8.22\left(\mathrm{~m}, 4 \mathrm{H}, \mathrm{C}_{6} \mathrm{H}_{4}\right), 9.30,10.27\left(2 \mathrm{~s}, 2 \mathrm{H}, \mathrm{NH}_{2}\right), 11.52(\mathrm{~s}$, $\left.1 \mathrm{H}, \mathrm{N}^{+} \mathrm{H}\right)$; EI-MS $\mathrm{m} / \mathrm{z}: 291\left(\mathrm{M}^{+}\right)$. Anal. calcd for $\mathrm{C}_{14} \mathrm{H}_{18} \mathrm{ClN}_{3} \mathrm{O}_{2} \mathrm{~S}$ : C 51.29, H 5.53, N 12.82; found C 51.35, $\mathrm{H} 5.55, \mathrm{~N} 12.80$.

4-叔丁基-6-(3,4-二甲氧基苯基)-2-氨基-1,3-噻嗪盐 酸盐(1i): 收率 42\%. m.p. 191 193 ${ }^{\circ} \mathrm{C} ;{ }^{1} \mathrm{H}$ NMR (400 $\left.\mathrm{MHz}, \mathrm{CDCl}_{3}\right) \delta: 1.29$ (s, 9H, $\left.\mathrm{CH}_{3} \times 3\right), 3.88\left(\mathrm{~s}, 6 \mathrm{H}, \mathrm{CH}_{3}\right.$ ), $5.00(\mathrm{~d}, J=5.6 \mathrm{~Hz}, 1 \mathrm{H}$, 噻嗪环 $5-\mathrm{H}), 5.19(\mathrm{~d}, J=5.6 \mathrm{~Hz}$, $1 \mathrm{H}$, 噻嗪环 $6-\mathrm{H}), 6.85 \sim 6.92\left(\mathrm{~m}, 3 \mathrm{H}, \mathrm{C}_{6} \mathrm{H}_{3}\right), 9.33,10.09$ $\left(2 \mathrm{~s}, 2 \mathrm{H}, \mathrm{NH}_{2}\right), 11.40\left(\mathrm{~s}, 1 \mathrm{H}, \mathrm{N}^{+} \mathrm{H}\right) ;{ }^{13} \mathrm{C}$ NMR $(100 \mathrm{MHz}$, $\left.\mathrm{CDCl}_{3}\right) \delta: 28.08\left(3 \mathrm{C}, \mathrm{CH}_{3} \times 3\right), 35.1\left(1 \mathrm{C}, \mathrm{CH}_{3} \times 3\right), 56.0$, $56.1\left(2 \mathrm{C}, \mathrm{OCH}_{3} \times 2\right), 43.0(1 \mathrm{C}$, 噻嗪环 6-C), $100.7(1 \mathrm{C}$, 噻嗪环 5-C), 110.9, 111.5 (2C, $\left.\mathrm{C}_{6} \mathrm{H}_{3}-2,5-\mathrm{C}\right), 120.4$ (1C, $\mathrm{C}_{6} \mathrm{H}_{3}-6-\mathrm{C}$ ), 130.1 (1C, $\mathrm{C}_{6} \mathrm{H}_{3}-1-\mathrm{C}$ ), 146.7 (1C, 噻嗪环 4-C), 149.4, 149.6 (2C, $\left.\mathrm{C}_{6} \mathrm{H}_{3}-3,4-\mathrm{C}\right), 167.8$ (1C, 噻嗪环 4-C); EI-MS $m / z$ : $306\left(\mathrm{M}^{+}\right)$. Anal. calcd for $\mathrm{C}_{16} \mathrm{H}_{23} \mathrm{ClN}_{2}$ $\mathrm{O}_{2} \mathrm{~S}$ : C 56.05, H 6.76, N 8.17; found C 55.94, H 6.78, N 8.19 .

4-叔丁基-6-(4-甲氧基苯基)-2-氨基-1,3-噻嗪盐酸盐 (1j): 收率 77\%. m.p. $167 \sim 170{ }^{\circ} \mathrm{C} ;{ }^{1} \mathrm{H}$ NMR $(400 \mathrm{MHz}$, $\left.\mathrm{CDCl}_{3}\right) \delta: 1.33\left(\mathrm{~s}, 9 \mathrm{H}, \mathrm{CH}_{3} \times 3\right), 3.87\left(\mathrm{~s}, 3 \mathrm{H}, \mathrm{CH}_{3}\right), 5.15(\mathrm{~d}$, $J=5.6 \mathrm{~Hz}, 1 \mathrm{H}$, 噻嗪环 $5-\mathrm{H}), 5.26(\mathrm{~d}, J=5.6 \mathrm{~Hz}, 1 \mathrm{H}$, 噻 嗪环 6-H), 6.90 (d, $\left.J=7.6 \mathrm{~Hz}, 2 \mathrm{H}, \mathrm{C}_{6} \mathrm{H}_{4}-3,5-\mathrm{H}\right), 7.32$ (d, $\left.J=7.6 \mathrm{~Hz}, 2 \mathrm{H}, \mathrm{C}_{6} \mathrm{H}_{4}-2,6-\mathrm{H}\right), 8.24,10.21\left(2 \mathrm{~s}, 2 \mathrm{H}, \mathrm{NH}_{2}\right)$, $11.55\left(\mathrm{~s}, 1 \mathrm{H}, \mathrm{N}^{+} \mathrm{H}\right)$. Anal. calcd for $\mathrm{C}_{15} \mathrm{H}_{21} \mathrm{ClN}_{2} \mathrm{OS}: \mathrm{C}$ 57.59, H 6.77, N 8.95; found C 57.39, H 6.79, N 8.93.

4-叔丁基-6-(2-氯苯基)-2-氨基-1,3-噻嗪盐酸盐(1k): 收率 65\%. m.p. $149 \sim 150{ }^{\circ} \mathrm{C} ;{ }^{1} \mathrm{H}$ NMR (400 MHz, $\left.\mathrm{CDCl}_{3}\right)$ $\delta: 1.33\left(\mathrm{~s}, 9 \mathrm{H}, \mathrm{CH}_{3} \times 3\right), 5.19(\mathrm{~d}, J=5.6 \mathrm{~Hz}, 1 \mathrm{H}$, 噻嗪环 $5-\mathrm{H}), 5.36(\mathrm{~d}, J=6.0 \mathrm{~Hz}, 1 \mathrm{H}$, 噻嗪环 $6-\mathrm{H}), 7.27 \sim 7.43$ $\left(\mathrm{m}, 4 \mathrm{H}, \mathrm{C}_{6} \mathrm{H}_{4}\right), 9.03,10.23\left(2 \mathrm{~s}, 2 \mathrm{H}, \mathrm{NH}_{2}\right), 11.59(\mathrm{~s}, 1 \mathrm{H}$, $\mathrm{N}^{+} \mathrm{H}$ ); EI-MS $m / z: 280\left(\mathrm{M}^{+}\right)$. Anal. calcd for $\mathrm{C}_{14} \mathrm{H}_{18} \mathrm{Cl}_{2^{-}}$ $\mathrm{N}_{2} \mathrm{~S}$ : C 53.00, H 5.72, N 8.83; found C 52.85, H 5.75, N 8.81 .

4-叔丁基-6-(4-氯苯基)-2-氨基-1,3-噻嗪盐酸盐(11): 收率 87\%. m.p. $228 \sim 230{ }^{\circ} \mathrm{C} ;{ }^{1} \mathrm{H} \mathrm{NMR}\left(400 \mathrm{MHz}, \mathrm{CDCl}_{3}\right)$ $\delta: 1.23\left(\mathrm{~s}, 9 \mathrm{H}, \mathrm{CH}_{3} \times 3\right), 5.05(\mathrm{~d}, J=6.0 \mathrm{~Hz}, 1 \mathrm{H}$, 噻溙环 5-H), 5.17 (d, $J=6.0 \mathrm{~Hz}, 1 \mathrm{H}$, 噻嗪环 6-H), 7.26 (d, $J=$ $\left.6.8 \mathrm{~Hz}, 2 \mathrm{H}, \mathrm{C}_{6} \mathrm{H}_{4}-2,6-\mathrm{H}\right), 7.35\left(\mathrm{~d}, J=6.8 \mathrm{~Hz}, 2 \mathrm{H}, \mathrm{C}_{6} \mathrm{H}_{4^{-}}\right.$ 3,5-H), 9.74, $9.99\left(2 \mathrm{~s}, 2 \mathrm{H}, \mathrm{NH}_{2}\right), 11.30\left(\mathrm{~s}, 1 \mathrm{H}, \mathrm{N}^{+} \mathrm{H}\right)$; ESI-MS $m / z$ : $292\left(\mathrm{M}^{+}\right)$. Anal. calcd for $\mathrm{C}_{14} \mathrm{H}_{18} \mathrm{Cl}_{2} \mathrm{~N}_{2} \mathrm{~S}: \mathrm{C}$ 53.00, H 5.72, N 8.83; found C 52.93, H 5.74, N 8.81.

4-叔丁基-6-(2,4-二氯苯基)-2-氨基-1,3-噻嗪盐酸盐 (1m): 收率 52\%. m.p. $105{ }^{\circ} \mathrm{C} ;{ }^{1} \mathrm{H}$ NMR $(400 \mathrm{MHz}$, $\left.\mathrm{CDCl}_{3}\right) \delta: 1.32\left(\mathrm{~s}, 9 \mathrm{H}, \mathrm{CH}_{3} \times 3\right), 5.17(\mathrm{~d}, J=5.2 \mathrm{~Hz}, 1 \mathrm{H}$, 噻嗪环 $5-\mathrm{H}), 5.30(\mathrm{~d}, J=4.4 \mathrm{~Hz}, 1 \mathrm{H}$, 噻嗪环 $6-\mathrm{H}), 7.20$ $7.44\left(\mathrm{~m}, 3 \mathrm{H}, \mathrm{C}_{6} \mathrm{H}_{3}\right), 9.28,10.20\left(2 \mathrm{~s}, 2 \mathrm{H}, \mathrm{NH}_{2}\right), 11.54$ (s, $\left.1 \mathrm{H}, \mathrm{N}^{+} \mathrm{H}\right)$; EI-MS $m / z: 315\left(\mathrm{M}^{+}\right)$. Anal. calcd for $\mathrm{C}_{14} \mathrm{H}_{17} \mathrm{Cl}_{3} \mathrm{~N}_{2} \mathrm{~S}$ : C 47.81, H 4.87, N 7.96; found C 47.64, H 4.89, N 7.94 .

4-叔丁基-6-(2-甲氧基苯基)-2-氨基-1,3-噻嗪盐酸盐 (1n): 收率 61\%. m.p. $140 \sim 142{ }^{\circ} \mathrm{C}$; ${ }^{1} \mathrm{H}$ NMR $(400 \mathrm{MHz}$, $\left.\mathrm{CDCl}_{3}\right) \delta: 1.33\left(\mathrm{~s}, 9 \mathrm{H}, \mathrm{CH}_{3} \times 3\right), 3.82\left(\mathrm{~s}, 3 \mathrm{H}, \mathrm{OCH}_{3}\right), 5.17$ (d, $J=6.4 \mathrm{~Hz}, 1 \mathrm{H}$, 噻嗪环 $5-\mathrm{H}), 5.26(\mathrm{~d}, J=6.4 \mathrm{~Hz}, 1 \mathrm{H}$, 噻嗪环 6- $\mathrm{H}), 6.88\left(\mathrm{~d}, J=7.8 \mathrm{~Hz}, 2 \mathrm{H}, \mathrm{C}_{6} \mathrm{H}_{4}-3-\mathrm{H}\right), 7.30$ (t, $\left.J=7.6 \mathrm{~Hz}, 2 \mathrm{H}, \mathrm{C}_{6} \mathrm{H}_{4}-4-\mathrm{H}\right), 6.94$ (t, $J=7.8 \mathrm{~Hz}, 2 \mathrm{H}$, $\left.\mathrm{C}_{6} \mathrm{H}_{4}-5-\mathrm{H}\right), 7.13$ (d, $\left.J=7.6 \mathrm{~Hz}, 2 \mathrm{H}, \mathrm{C}_{6} \mathrm{H}_{4}-6-\mathrm{H}\right), 7.65,10.45$ $\left(2 \mathrm{~s}, 2 \mathrm{H}, \mathrm{NH}_{2}\right), 11.75\left(\mathrm{~s}, 1 \mathrm{H}, \mathrm{N}^{+} \mathrm{H}\right)$; EI-MS $m / z$ : $276\left(\mathrm{M}^{+}\right)$. Anal. calcd for $\mathrm{C}_{15} \mathrm{H}_{21} \mathrm{ClN}_{2} \mathrm{OS}$ : C 57.59, H 6.77, N 8.95; found C 57.47, H 6.79, N 8.94.

4-叔丁基-6-[3-(三氟甲基)苯基]-2-氨基-1,3-噻嗪盐 酸盐(10): 收率 52\%. m.p. $186 \sim 187{ }^{\circ} \mathrm{C} ;{ }^{1} \mathrm{H}$ NMR (400 $\left.\mathrm{MHz}, \mathrm{CDCl}_{3}\right) \delta: 1.29\left(\mathrm{~s}, 9 \mathrm{H}, \mathrm{CH}_{3} \times 3\right), 5.16,5.19[2 \mathrm{~s}, 2 \mathrm{H}$, 噻嗪环 5,6- $\mathrm{H}_{2}$ ] $, 7.52 \sim 7.66\left(\mathrm{~m}, 4 \mathrm{H}, \mathrm{C}_{6} \mathrm{H}_{4}\right), 9.78,10.11(2 \mathrm{~s}$, $\left.2 \mathrm{H}, \mathrm{NH}_{2}\right), 11.32\left(\mathrm{~s}, 1 \mathrm{H}, \mathrm{N}^{+} \mathrm{H}\right)$; EI-MS $m / z: 314\left(\mathrm{M}^{+}\right)$. Anal. calcd for $\mathrm{C}_{15} \mathrm{H}_{18} \mathrm{ClF}_{3} \mathrm{~N}_{2} \mathrm{~S}$ : C 51.35, H 5.17, N 7.98; found C 51.23, H 5.19, N 7.99.

4-叔丁基-6-苯基-2-氨基-1,3-噻嗪盐酸盐(1p): 收率 $53 \%$. m.p. $137 \sim 140{ }^{\circ} \mathrm{C} ;{ }^{1} \mathrm{H}$ NMR (400 MHz, $\left.\mathrm{CDCl}_{3}\right) \delta$ : $1.27\left(\mathrm{~s}, 9 \mathrm{H}, \mathrm{CH}_{3} \times 3\right), 5.02(\mathrm{q}, J=4.8 \mathrm{~Hz}, 1 \mathrm{H}$, 噻嗪环 $5-\mathrm{H}), 5.20$ (q, $J=4.8 \mathrm{~Hz}, 1 \mathrm{H}$, 噻嗪环 $6-\mathrm{H}$ ), $7.33 \sim 7.38$ $\left(\mathrm{m}, 5 \mathrm{H}, \mathrm{C}_{6} \mathrm{H}_{5}\right), 9.66,10.00\left(2 \mathrm{~s}, 2 \mathrm{H}, \mathrm{NH}_{2}\right), 11.32(\mathrm{~s}, 1 \mathrm{H}$, $\mathrm{N}^{+} \mathrm{H}$ ); EI-MS $m / z$ : $246\left(\mathrm{M}^{+}\right)$. Anal. calcd for $\mathrm{C}_{14} \mathrm{H}_{19} \mathrm{Cl}-$ $\mathrm{N}_{2} \mathrm{~S}$ : C 59.45, H 6.77, N 9.90; found C 59.50, H 6.79, N 9.91 .

\section{3 生物活性测试}

\subsection{1 神经氨酸酶抑制活性测试}

乙酰神经氨酸(MUNANA) 是神经氨酸酶的特异性 底物, 在神经氨酸酶作用下产生的代谢产物在 $360 \mathrm{~nm}$ 波长激发下, 可以产生 $450 \mathrm{~nm}$ 荧光, 荧光强度的变化可 以灵敏地反应神经氨酸酶活性 ${ }^{[27 ~ 29]}$.

在酶反应体系中, 一定浓度待测样品与感病毒 NA 悬浮于反应缓冲液中 ( $\mathrm{pH}$ 6.5), 加入苂光底物 MUNANA 
启动反应体系, $37^{\circ} \mathrm{C}$ 罦育 $60 \mathrm{~min}$ 后, 加反应终止液终止 反应. 在激发波长 $360 \mathrm{~nm}$ 和发射波长为 $450 \mathrm{~nm}$ 的参数 条件下, 测定荧光强度值. 根据荧光强度的减少量计算 化合物对 NA 活性的抑制率. 分别评价了每个样品在反 应体系中浓度为 $40 \mu \mathrm{g} / \mathrm{mL}$ 对 $\mathrm{NA}$ 的抑制活性.

$$
\text { 抑制率 }=\frac{(\text { 酶值一空白值 })-(\text { 实验值－空白值 })}{\text { 酶值－空白值 }} \times 100 \%
$$

\subsubsection{5-羟色胺重摄取抑制活性测试}

SERT 是 5-HT 重摄取相关的重要靶点, 现有的一线 抗抑郁药物几乎均为 SERT 抑制剂. 荧光染料 ASP 是一 种具有神经毒性的化合物, 可以与单胺类的转运体结合 进入细胞内, 并发出黄色荧光. 当存在其他可与转运体 结合化合物同 ASP 进行竞争时, 进入细胞内的 ASP 数 量减少, 黄色苂光减弱, 可通过比较荧光强度的变化笁 选作用于 SERT 的重摄取抑制剂. RBL-2H3 细胞是一种 可以分泌组胺和 5-HT 的细胞株, 该细胞是永生化的肥 大细胞 ${ }^{[30]}$, 可以量子释放前述的递质. 并且具有 SERT 可以进行 5-HT 重摄取. CACO-2 细胞是来源于人小肠的 腺癌细胞株, 经过培养分化该细胞可以表达多种转运体 和受体，包括 SERT 和腺苷受体等.

将 RBL 细胞悬液加入 96 孔板, 待细胞贴壁生长 10 $\mathrm{h}$ 以上, 加入待笁选的样品一段时间, 再按照一定浓度 加入 ASP, 洗去未进入细胞的染料, 在激发波长 475 $\mathrm{nm}$, 发射波长 $605 \mathrm{~nm}$ 处读数, 进行结果统计. 将 CACO-2 细胞加入 96 孔黑色底透明培养板, 待其分化, 生长 6 7 日可见细胞界限不清, 生长微线毛, 加入待笁 选的供试品一段时间, 再按照一定浓度加入 ASP, 在激 发波长 $475 \mathrm{~nm}$, 发射波长 $605 \mathrm{~nm}$ 处读数, 进行结果统 计. 分别评价了样品在 $10 \mu \mathrm{g} / \mathrm{mL}$ 时对 SERT 的抑制活 性.

$$
\text { 抑制率 }=\frac{\text { 对照值一样品值 }}{\text { 对照值一空白值 }} \times 100 \%
$$

\section{References}

[1] Sun, C. W.; Zhang, X. D.; Huang, H.; Zhou, P. Bioorg. Med. Chem. 2006, 14, 8574.

[2] Baz, M.; Abed, Y.; Boivin, G. Antiviral Res. 2007, 74, 159.

[3] Baranovich, T.; Webster, R. G.; Govorkova, E. A. Curr. Opin. Virol. 2011, 1, 574.

[4] Collins, P. J.; Haire, L. F.; Lin, Y. P. Nature 2008, 453, 1258.
[5] Abed, Y.; Nehm, B.; Baz, M.; Boivin, G. Antiviral Res. 2008, 77, 163.

[6] Mitrasinovic, P. M. Curr. Drug. Targets 2010, 11, 315.

[7] Chand, P.; Kotian, P. L.; Morris, P. E.; Bantia, S.; Walshb, D. A.; Babu, Y. S. Bioorg. Med. Chem. 2005, 13, 2665.

[8] You, Q. D. Pharmaceutical Chemistry, Chemical Industry Press, Beijing, 2004, pp. 145 149 (in Chinese). (尤启东, 药物化学, 化学工业出版社, 北京, 2004, pp. 145 149.)

[9] Christmas, D. M.; Potokar, J. P.; Davies, S. J. C. Neurobiol. Dis. Treat. 2011, 7, 431

[10] Akhondzadeh, S.; Jafari, S.; Raisi, F.; Nasehi, A. A.; Ghoreishi, A.; Salehi, B.; Mohebbi-Rasa, S.; Raznahan, M. Depression Anxiety 2009, 26, 607.

[11] Francesc. A. ACS Chem. Neurosci. 2013, 4, 5 .

[12] Lynette. C. D.; Wouter. K.; Nathan. C. M. ACS Chem. Neurosci. 2013, 4, 16.

[13] Hector, H.; Sylvain, B.; Leticia, Q.; Estibaliz, S.; Aurelio, O. Tetrahedron Lett. 2006, 47, 1153.

[14] Temple, C.; Wheeler, G. P.; Comber, R. N.; Elliot, R. D.; Montgomery, J. A. J. Med. Chem. 1983, 26, 1614.

[15] Singh, U. P.; Pathak, M.; Dubey, V.; Bhat, H. R.; Gahtori, P.; Singh, R. K. Chem. Biol. Drug Des. 2012, 80, 572.

[16] Koketsu, M.; Tanaka, K.; Takenaka, Y.; Kwong, C. D.; Ishihara, H. Eur. J. Pharm. Sci. 2002, 15, 307.

[17] Gazic, I.; Bosak, A.; Sinko, G.; Vinkovic, V.; Kovarik, Z. Anal. Bioanal. Chem. 2006, 385, 1513.

[18] Handley, D. A.; McCullough, J. R.; Crowther, S. D.; Morley, J. Chirality 1998, 10, 262.

[19] Landon, M. R.; Amaro, R. E.; Baron, R. Chem. Biol. Drug Des. 2008, $71,106$.

[20] Grienke, U.; Schmidtke, M.; Kirchmair, J. J. Med. Chem. 2010, 53, 778.

[21] An, J. H.; Lee, D. C. W.; Law, A. H. J. Med. Chem. 2009, 52, 2667.

[22] Wang, Y.; Hu, A. X.; Cao, G.; Li, G. X.; Zhang, J. Y.; Xia, L.; Ou, X. M.; Xu, J. B. Chin. J. Org. Chem. 2008, 28, 443 (in Chinese). (王宇, 胡艾希, 曹高, 李国希, 张建宇, 夏林, 欧晓明, 徐建兵, 有机化学, 2008, 28, 443.)

[23] Wu, T. Q.; Xia, L.; Hu, A. X.; Ye, J. Acta. Crystallogr. 2009, E65, 0635.

[24] Xia, L.; Hu, A. X.; Cao, G..; Wang, Y.; Ye, J. Chin. J. Struct. Chem. 2009, 28, 33 (in Chinese).

(夏林, 胡艾希, 曹高, 王宇, 叶姣, 结构化学, 2009, 28, 33.)

[25] Hu, A. X.; Xia, L. CN 101440072, 2009 [Chem. Abstr. 2009, 151, 78128].

[26] Hu, A. X.; Xia, L. CN 101570521, 2009 [Chem. Abstr. 2009, 151, 571117].

[27] Liu, A. L.; Cao, H. P.; Du, G. H. Sci. China, Ser. C 2005, 48, 1

[28] Laver, W. G.; Goleman, P. M.; Hinshaw, V. S.; Air, G. M. Virology 1984, 137, 314.

[29] Liu, Y.; Zhang, L.; Gong, J. Z.; Fang, H.; Liu, A. L.; Du, G. H.; Xu, W. F. J. Enzyme Inhib. Med. Chem. 2011, 26, 506.

[30] Kawana. S.; Kato. Y.; Omi. T. Clin. Exp. Dermatol. 2010, 35, 835.

(Qin, X.) 